\title{
Primary Uterine Lymphoma: A Case Report
}

In Joo Cheong, MD

Se Hyun Kim, MD

Cheol Min Park, MD

\section{Index terms:}

Uterus, lymphoma

Uterus, magnetic resonance imaging

Uterus, neoplasm

Korean J Radiol 2000 ; 1 :223-225

Received January 17, 2000; accepted after revision September 13, 2000.

All authors: Department of Diagnostic Radiology, Korea University Guro Hospital.

Address reprint requests to: Cheol Min Park, MD, Department of Diagnostic Radiology, Korea University Guro Hospital, 80, Guro-dong, Guro-gu, Seoul 152-050, Korea.

Telephone: (822) 818-6183

Fax: (822) 863-9282

e-mail: cminpark@korea.ac.kr
Primary lymphoma of the uterus is a rare disease, the reported characteristic MR imaging findings being homogeneous intermediate signal intensity of the indistinct mass on T1- and T2-weighted images, and the preservation of endometrial lining and uterine architecture. We report a case of primary uterine lymphoma which showed tumoral necrosis, endometrial disruption and diffuse anterior vaginal wall involvement.

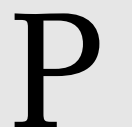

rimary lymphoma arising from the female genital tract is extremely rare. Twenty-five percent of malignant lymhomas arise at extranodal sites and in only 1 percent of women with extranodal tumors is the genital tract diseased (1). If malignant lymphoma is widely disseminated, the uterine corpus, cervix and vagina are frequently involved. Because of its rarity and the poor yield of cervical cytology due to the nature of its infiltration of the muscular layer and rare mucosal ulceration, diagnosis of the condition is not easy. With appropriate treatment, the prognosis is relatively good, however, and exact diagnosis and evaluation of the extent of the disease is therefore essential $(1,2)$. It has been reported that in spite of diffuse involvement, the preservation of uterine architecture, normal endometrial enhancement and tumoral homogeneity are the characteristic MR imaging features of uterine and cervical lymphoma (3-7). To our knowledge, however, the literature does not include descriptions of tumoral necrosis and destruction of the endometrial lining. We present a case of primary uterine lymphoma in which MR imaging revealed internal necrosis and destruction of the endometrial lining of a diffusely enlarged uterus.

\section{CASE REPORT}

A 62-year-old woman experienced lower abdominal pain and vaginal bleeding lasting for 20 days. She had neither a previous history nor general symptoms of lymphoma. Her CA15-3 level was $15.6 \mathrm{U} / \mathrm{mL}$, a-FP was $1.9 \mathrm{ng} / \mathrm{mL}$, and CEA was 0.6 $\mathrm{ng} / \mathrm{mL}$. Physical examination revealed marked enlargement of the uterus, though a smear test proved negative.

US demonstrated lobulated uterine enlargement, with a focal anechoic area (Fig. 1A). Sagittal MR images of the pelvis showed diffuse enlargement of the uterus and cervix, both of which exhibited homogeneous intermediate signal intensity on T1weighted image (TR [msec] / TE [msec]: 700/20) and T2-weighted image (TR/TE: 2000/90) and contained an area of focal high-signal intensity, as revealed by both these modalities. Diffuse anterior vaginal wall involvement was also noted, and neither the junctional zone nor normal cervical stroma were preserved (Figs. 1B, C). Gadolinium-enhanced MR images showed relatively uniform homogeneous enhance- 
ment of the uterus, but no endometrial enhancement (Fig. ID). Pelvic lymphadenopathy was not observed. Needle biopsy of the vagina indicated the presence of a benign fibromuscular tumor, and trans-abdominal hysterectomy and bilateral salpingo-oophorectomy were performed.

A gross specimen showed extensive necrosis of the uterus, and microscopic examination of the mass demonstrated diffuse infiltration of the myometrium, endometrium and endocervix, as well as the parametrium, vagina and adnexa, by atypical high-grade lymphoblastic lymphoma cells. Bone marrow biopsy of the sternum indicated $65 \%$ infiltration by tumor cells. High-grade lymphoblastic lymphoma of the uterus with bone marrow invasion (Ann Arbor Stage IV) was diagnosed.

\section{DISCUSSION}

Compared with lymphoma of the ovary, malignant lymphoma of the uterus and cervix occurs in patients of very different ages, the median - particularly if the prognosis is relatively good - being the fourth decade. For prediction of a patient' $s$ survival, the stage already reached by the disease is the most important factor $(1,2)$. Even in patients with extensive local disease, FIGO stage II and III, the prognosis is good if treatment with radiation therapy and/or chemotherapy is undertaken $(1,2,6)$. Evaluation of the extent of the disease by imaging studies, especially the matter of whether or not the lesion involves adjacent organs such as the vagina, urethra and bladder, is important

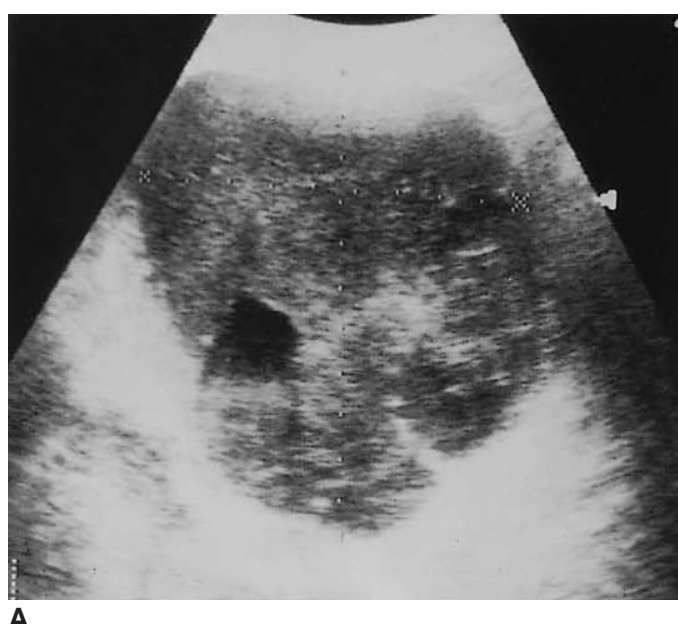

A

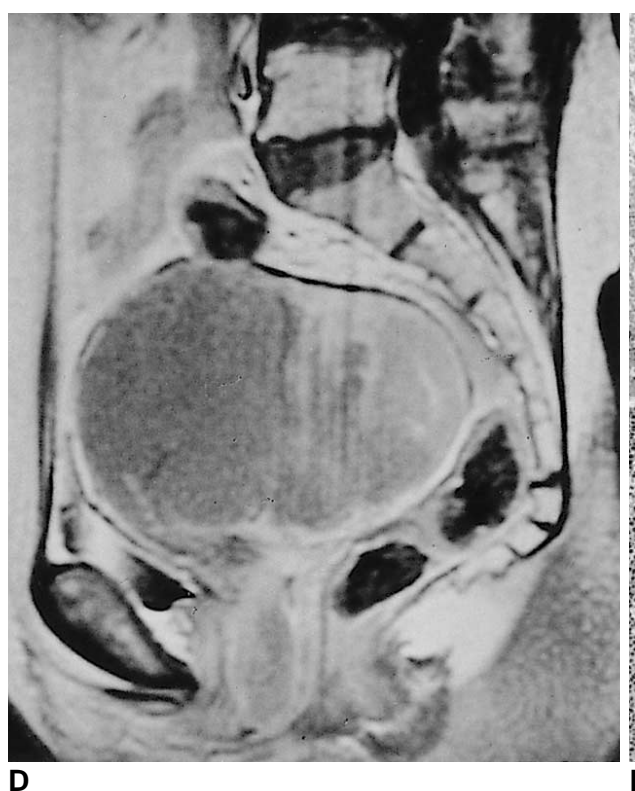

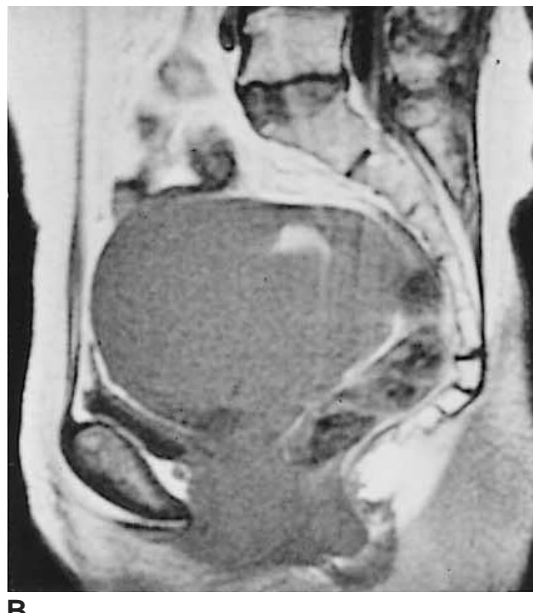

B

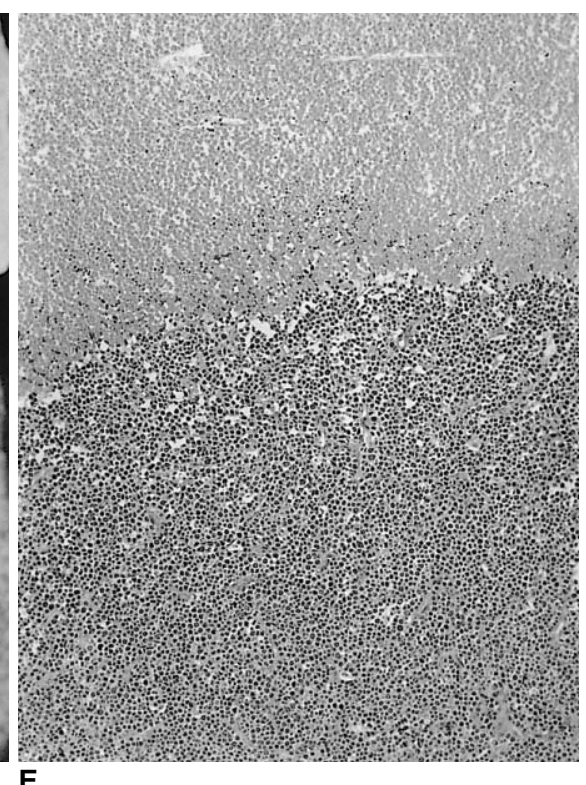

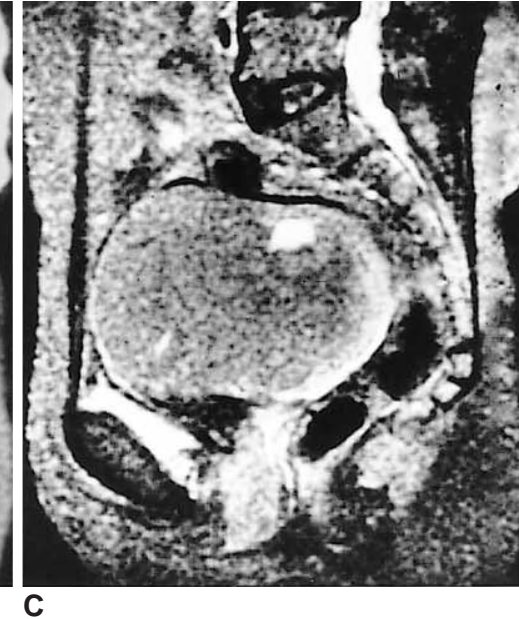

Fig. 1. Primary lymphoma in a 62-yearold woman.

A. US reveals hypoechoic lobulated uterine enlargement, with an anechoic portion. An echogenic endometrial line is not present.

B. Sagittal T1-weighted image shows homogeneous diffuse enlargement of the uterus, with an area of focal high signal intensity. The anterior vaginal wall is thickened.

C. Sagittal T2-weighted image shows a low signal intensity uterus containing a high signal intensity area representing hemorrhagic necrosis. The zonal anatomy of the endometrium, myometrium and endocervix is not preserved, and the junctional zone is indistinct.

D. T1-weighted image after gadolinium administration reveals inhomogeneous, moderate enhancement of the uterus and anterior vaginal wall.

E. On photomicrograph the border between the myometrium and endometrium is blurred by the infiltration of lymphoid tumor cells, and the tumor shows extensive necrosis ( $H \& E)$. 
in deciding the method of treatment. For definitive diagnosis of the disease, large incisional or excisional biopsy is preferable to cervical cytology $(1,2,7)$.

Uterine and cervical lymphoma showed variable features, from diffuse enlargement of the uterus to localized masses. According to a clinicopathologic analysis of 27 cases of gynecologic lymphoma by Harris and Scully (2), 44\% of these showed diffuse enlargement without mucosal abnormality and $41 \%$ showed variable mass-like findings such as multinodular mass, endocervical polyp and submucosal mass. In two cases, only an endometrial lesion was present. Lymphoma of the vagina however, always produces ill-defined wall thickening or indentation (2). Lymphoma can mimic cervical or endometrial carcinoma or degeneratig myoma, manifesting on T2-weighted images as a mass with increased signal intensity. Unlike degenerative myoma and cervical or endometrial carcinoma, gynecologic lymphoma is not sharply demarcated and shows moderate and uniform enhancement (3-5). Due to the fact that the condition is infiltrative, without causing stromal reaction, architectural preservation and an intact endometrium and/or junctional zone have been reported to be characteristic features of lymphoma $(4,6,7)$. In older, postmenopausal women, the junctional zone becomes thinner. The endometrium and myometrium are infiltrated by poorly differentiated lymphoid cells and this causes endometrial destruction and an indistinct junctional zone. Architectural effacement of extranodal tissue, which can usually be appreciated grossly, provides additional support for the histologic diagnosis of lymphoma (8).

Tumoral necrosis of lymphoma is not frequent but can occur when the mass is large enough. Loss of endometrial enhancement and poor differentation between the en- dometrium and myometrium correlated closely with the microscopic findings, which demonstrated diffuse infiltration of the endometrium and myometrium by high-grade lymphoblastic lymphoma, with a blurred margin between the two layers.

In conlusion, we believe that a diagnosis of lymphoma should be considered when a markedly enlarged uterus a) loses its normal uterine zonal architecture, b) shows low to intermediate signal intensity on both T1- and T2-weighted images, or c) shows poor and inhomogeneous enhancement even if areas of necrosis and endometrial disruption are present.

In addition, for confirmative diagnosis, tissue biopsy is essential. Diffuse vaginal wall thickening can be an ancillary feature of gynecologic lymphoma.

\section{References}

1. Komaki R, Cox J, Hansen R, Gunn W, Greeberg M. Malignant lymphoma of the uterus and cervix. Cancer 1984;54:1699-1974

2. Harri NL, Scully RE. Malignant lymphoma and granulocytic sarcoma of the uterus and vagina. Cancer 1984;3:2530-2545

3. Kawakami S. Togashi K, Kojima N, Morikawa K, Mori T, Konishi J. MR appearance of maliganant lymphoma of the uterus. J Comput Assist Tomogr 1995;19:238-242

4. Yamamda I. Primary uterine lymphoma: MR imaging. AJR 1993;160:662-663

5. Dang HT, Terk HR, Colleti PM, Schlaerth J, Cutin J. Primary lymphoma of the cervix: MRI findings with gadolinium. Magn Reson lmaging 1991;9:941-944

6. Kimura I, Togashi K, Tsutsui K, et al. MR imaging of gynecologic lymphoma. J Comput Assist Tomogr 1991;15:550-501

7. Kim YS, Joh BH, Cho OK, Rhim HC. MR imaging of primary uterine lymphoma. Abdom Imaging 1997;22:441-444

8. Stickler JG, Burgart LJ,Weiss LM. Classical Hodgkin's Disease. In Weiss LM, ed. Pathology of lymph nodes: Contemporary issues in surgical patholgy: Churchill Livingstone, 1996:1-214 